\title{
Phase solution analysis for the simultaneous dual frequency VLBI observations
}

\author{
Taehyun Jung $^{\text {1ab }}$, Bong Won Sohn ${ }^{a}$, Hideyuki Kobayashic ${ }^{c}$, Tetsuo Sasao ${ }^{d}$, \\ Tomoya Hirota $^{\mathrm{c}}$, Osamu Kameya ${ }^{\mathrm{c}}$, Yoon Kyung Choi ${ }^{\mathrm{e}}$, Hyun Soo Chung ${ }^{\mathrm{a}}$ \\ ${ }^{a}$ Korea Astronomy and Space Science Institute, Daejeon, Republic of Korea \\ ${ }^{b}$ University of Science and Technology, Daejeon, Republic of Korea \\ ${ }^{c}$ Mizusawa VERA Observatory, NAOJ, Mitaka, Tokyo, Japan \\ ${ }^{d}$ Ajou University, Suwon 442-749, Republic of Korea \\ ${ }^{e}$ Max-Planck-Institut für Radioastronomie, Bonn, Germany \\ E-mail: thjung@kasi.re.kr
}

\begin{abstract}
We present the results of the first simultaneous dual-frequency VLBI observation using VERA (VLBI Exploration of Radio Astrometry). This experiment is a pilot study to test the feasibility of multi-frequency phase referencing technique, which will be a main phase referencing method for KVN (Korean VLBI Network). A pair of bright continuum sources NRAO 512 at $22 \mathrm{GHz}$ and 3C 345 at $43 \mathrm{GHz}$ were simultaneously observed with dual beams of VERA, and the fringe phases obtained for the two sources were compared to monitor the phase fluctuation at the two different frequencies. The connected phase solutions clearly showed the non-dispersive characteristics of the neutral atmosphere at the observing frequencies. For the differential phases of the two sources, the Allan standard deviation shows the white phase noise behaviour up to the time scale of $\sim 1000 \mathrm{sec}$. These preliminary results demonstrate that the multi-frequency phase referencing technique, which will be implemented in KVN is a promising tools to remove the atmospheric phase fluctuation effectively.
\end{abstract}

The 9th European VLBI Network Symposium on

The role of VLBI in the Golden Age for Radio Astronomy and EVN Users Meeting

Bologna, Italy

September 23-26, 2008

\footnotetext{
$1 \quad$ Speaker
} 


\section{Introduction}

One of the main difficulties in mm-VLBI is the atmospheric phase fluctuation caused by a rapid variation of water vapour content, which results in sever coherence loss at high frequency ([1], [2], [3]). To compensate the atmospheric phase fluctuations effectively, several phase referencing methods have been proposed. Among these techniques, the multi-frequency phase referencing technique, in which fringe phases obtained at lower frequency are transferred to those at higher frequency to cancel out the atmospheric phase fluctuations, is expected to have great advantage in mm-VLBI [3]. For this reason, Korean VLBI Network (KVN) introduces the multi-frequency simultaneous receiving system for phase referencing, in which a target source can be observed simultaneously at four bands $(22,43,86$, and $129 \mathrm{GHz})$ ([4], [5], [6], [7]). In order to investigate the feasibility of the multi-frequency phase referencing technique for KVN, we carried out the simultaneous dual frequency VLBI observation with VERA (VLBI Exploration of Radio Astronomy).

\section{Observation}

VERA system is designed to observe simultaneously two different sources, one for a target and the other for a calibrator, at the same frequency with the so-called "dual-beam receiving system" ([8], [9]). Using VERA's dual-beam system, the atmospheric phase fluctuation was effectively removed by observing a target and a calibrator at the same time [10]. Usually VERA's dual-beam observes two sources (a target and a reference source) at the same frequency. In this experiment, however, we manually modified the receiving system so that we observe two sources at two different frequencies, 22 and $43 \mathrm{GHz}$. The first dual frequency observation in VERA was carried out on 2005 April 15 from UT 14:30 to UT 21:30 with the four VERA telescopes. We observed a bright BL Lac object NRAO 512 at $22 \mathrm{GHz}$ (beam A) and a bright quasar 3C 345 at $43 \mathrm{GHz}$ (beam B). The separation angle between the two sources is less than 0.5 degree and thus we can fairly assume that the atmospheric condition for both sources is almost same [2]. The bandwidth was $128 \mathrm{MHz}$ for each source and a single left-hand circular polarization was received. To calibrate the mechanical delay of dual beams in VERA, a noise source is usually injected into dual beam receivers during the observation of astronomical objects [11]. For this experiment, however, a noise source was not available because we observed two sources at two different frequencies. Therefore, we applied the manual phase calibration to remove the mechanical delay between the dual beams by using measured phases from high SNR fringes. The data reduction was performed with Astronomical Image Processing System (AIPS) package. The phase solutions were derived by the global fringe fitting procedure, FRING in AIPS. 


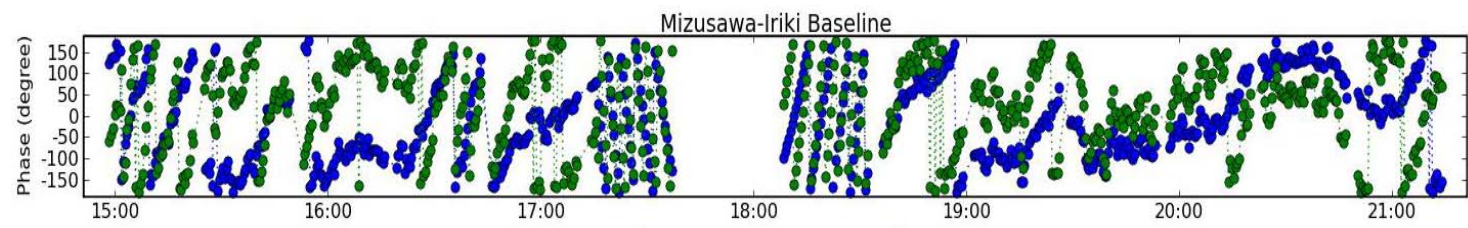

Mizusawa-Ogasawara Baseline
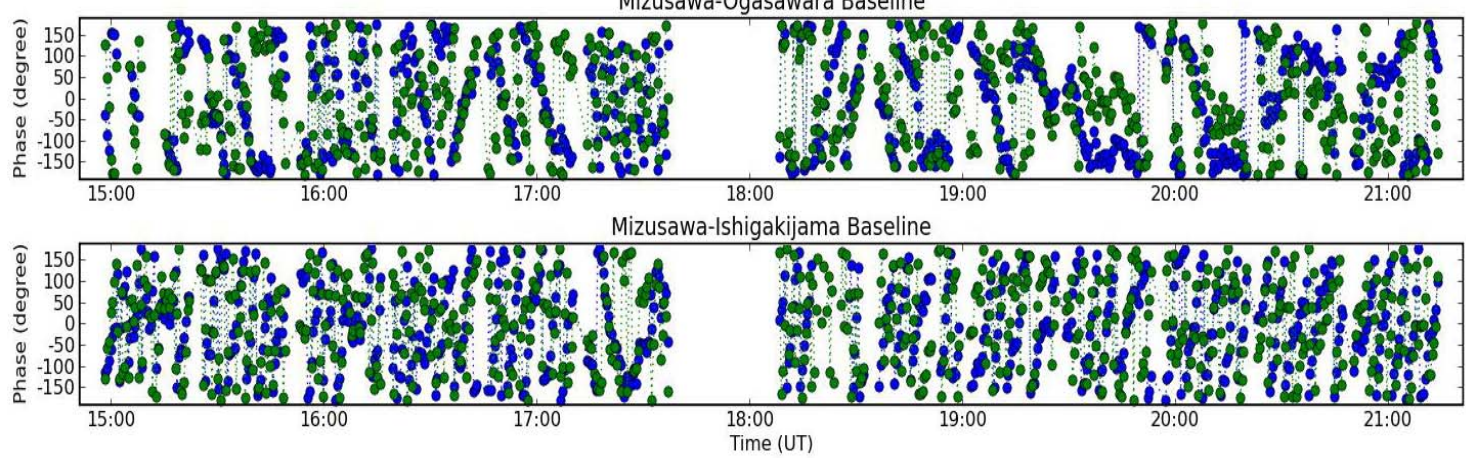

Figure 1. The phase solutions of $22 \mathrm{GHz}$ (blue dotted lines) and $43 \mathrm{GHz}$ (green dotted lines) at Mizusawa-Iriki (top), Mizusawa-Ogasawara (middle) and Mizusawa-Ishgakijima (bottom) baselines. Mizusawa was the reference antenna.

\section{Results}

Figure 1 shows the phase solutions of two continuum sources NRAO 512 (blue dotted lines) and 3C 345 (green dotted lines). The solution interval was 30 seconds and the reference antenna was set to Mizusawa. The phase variation was the most stable at the Mizusawa-Iriki $(1266 \mathrm{~km})$ baseline, whereas other solutions varied more rapidly (Mizusawa-Ogasawara: 1336 km, Mizusawa-Ishigakijima: $2270 \mathrm{~km}$ ).

After resolving $2 \pi$ ambiguities, the connected phases (top) and the differential phases (bottom) for Mizusawa-Iriki baseline are shown in figure 2. The left and right panels are the first half and the second half of the observation. It clearly appears that a tight correlation exists in the connected phase solutions at 22 and $43 \mathrm{GHz}$. This result implies that the $43 \mathrm{GHz}$ phase solution can be estimated from the $22 \mathrm{GHz}$. It also proves the non-dispersive characteristics of atmospheric phase delays,

$$
\delta \phi_{H}=\frac{v_{H}}{v_{L}} \delta \phi_{L}
$$

where $\delta \varphi_{\mathrm{H}}$ and $\delta \varphi_{\mathrm{L}}$ are the phase variations at high- and low- frequency and also $v_{\mathrm{H}}$ and $v_{\mathrm{L}}$ are the high- and low- observation frequency, respectively. The differential phase $\Delta \phi$ can be described as

$$
\Delta \phi=\phi_{43}-r \phi_{22}
$$

Here, $\phi_{22}$ and $\phi_{43}$ indicate the phase solutions at 22 and $43 \mathrm{GHz}$, respectively and $r$ is the frequency ratio: $r=v_{43 \mathrm{GHz}} / v_{22 \mathrm{GHz}} \sim 1.926$. If the phase calibration is perfect, the differential phases should be zero. Some residual phase errors remain, however, which may be attributed to 

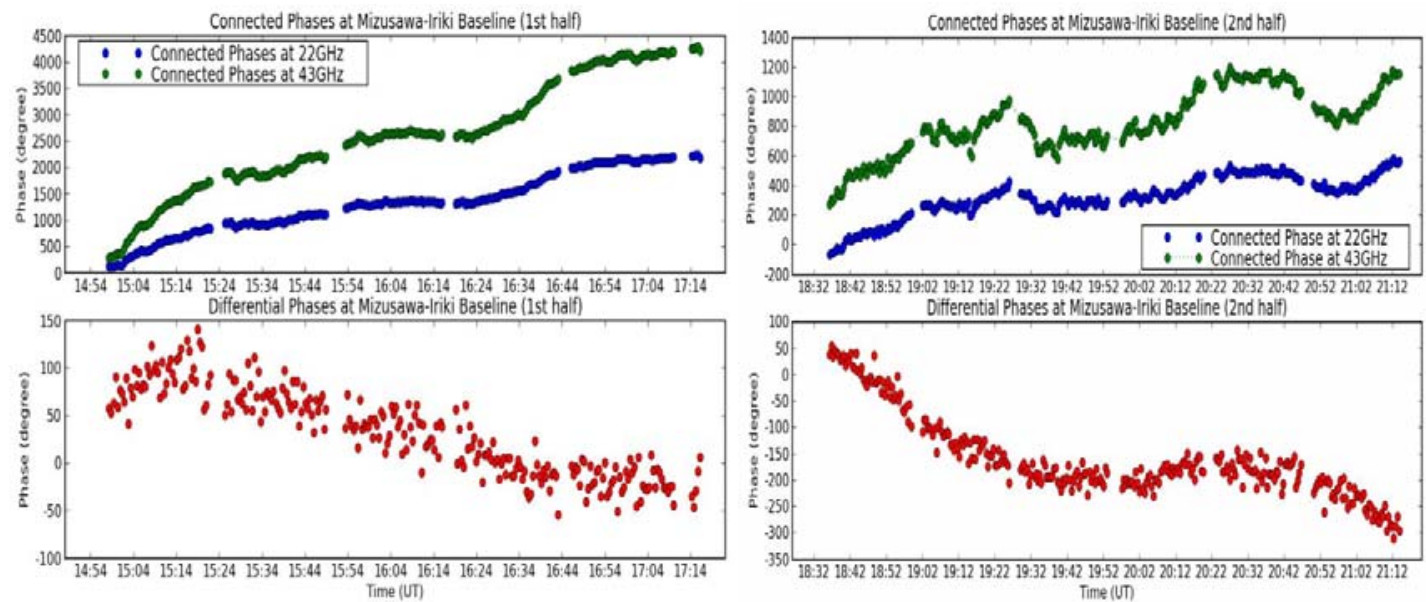

Figure 2. Top: The connected phase solutions of $22 \mathrm{GHz}$ (blue dotted lines) and $43 \mathrm{GHz}$ (green dotted lines) at Mizusawa-Iriki baseline. Bottom: The differential phases subtracted the scaled up phases of $22 \mathrm{GHz}$ by mutiplying the frequency ratio ( 1.926) from the original phases of $43 \mathrm{GHz}$. The left and right plots are the first half and the second half of observation.

the effects of source structure, instrumental phase drifts and/or the ionosphere. The differential phase variations are about 200 degrees and 360 degrees during the first half (140 minutes) and the second half (160 minutes) of observation.

To investigate the characteristics of phase variation, we calculated the Allan standard deviation for the differential phases (figure 3). The Allan standard deviation, $\sigma_{y}(\tau)$, can be calculated as follows [12],

$$
\sigma_{y}^{2}(\tau)=\frac{\left\langle[\phi(t+2 \tau)-2 \phi(t+\tau)+\phi(t)]^{2}\right\rangle}{8 \pi^{2} \nu_{0}^{2} \tau^{2}}
$$

where $\varphi$ is the observed phase, $\tau$ is time interval, $v_{0}$ is the observational frequency and the bracket \langle\rangle indicates the average over the whole samples. In figure 3, the Allan standard deviation of the differential phase between the time interval $30 \mathrm{sec}$ and $1000 \mathrm{sec}$ is showing almost white-phase noise, which is decreasing with $\tau^{-1}$. This implies that the phase calibration at higher frequency is feasible by using lower frequency phase solutions.

\section{Summary}

We have tested the feasibility of the phase solution transfer between $22 \mathrm{GHz}$ and $43 \mathrm{GHz}$ by the simultaneous dual frequency VLBI observation using VERA. The phase solutions showed a tight correlation between two frequencies and the Allan standard deviation of the differential phase solutions indicates that the multi-frequency phase referencing is achievable.

Asaki et al. [13] demonstrated that the high frequency phase delay (146 GHz) can be compensated by the lower frequency phase information $(19.5 \mathrm{GHz})$ with Paired Antenna Method (PAM). Middelberg et al. [14] also tested the feasibility of Fast Frequency Switching (FFS) method between the frequency of $14.5 \mathrm{GHz}$ and $86 \mathrm{GHz}$. Both of these 


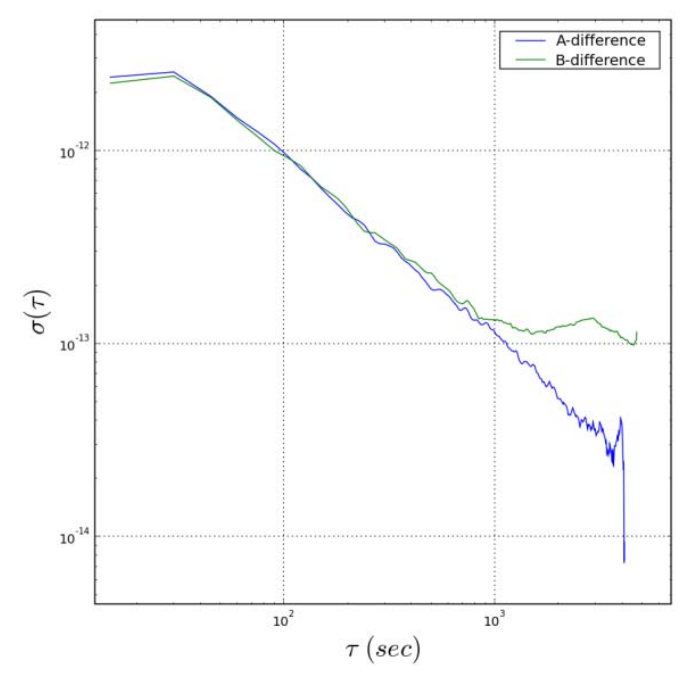

Figure 3. The Allan standard deviation of differential phases at Mizusawa-Iriki baseline. The differential phases of the first half and the second half are shown as blue line and green line, respectively.

methods have a spatial or timely incoherence in phase compensation. However, KVN is designed for multi-frequency observation of a target source, and hence further improvement in phase compensation is expected, especially for $86 \mathrm{GHz}$ or higher frequencies.

\section{References}

[1] A. E. E. Rogers, A. T. Moffet, D. C. Backer, J. M. Moran, 1984, Radio Science, 19, 1552

[2] Y. Asaki, et al., 1996, Radio Science, 31, 1615

[3] T. Sasao, 2003. New Technologies in VLBI, in ASP Conf. Ser. 306, ed. Y. C. Minh, 53

[4] Y. C. Minh et al., 2003. New Technologies in VLBI, in ASP Conf. Ser. 306, ed. Y. C. Minh, 373

[5] H. G. Kim et al., 2004, $7^{\text {th }}$ EVN Symposium, 281

[6] B. W. Sohn et al., 2006, $8^{\text {th }}$ EVN Symposium, 440

[7] S.-T. Han et al., 2008, International Journal of Infrared and Millimeter Waves 29, 69

[8] N. Kawaguchi et al., 2000, in Proceedings of SPIE 4015, Radio Telescope, ed. H. R. Butcher, 544

[9] H. Kobayashi et al., 2003, New Technologies in VLBI, in ASP Conf. Ser. 306, ed. Y. C. Minh, 367

[10] M. Honma et al., 2003, PASJ, 44, 57

[11] M. Honma et al., 2000, in Proceedings of SPIE 4015, Radio Telescope, ed. H. R. Butcher, 624

[12] A. R. Thompson, J. M. Moran, \& G. W. Swenson, Jr. 2001, Interferometry and Synthesis in Radio Astronomy, 2nd ed. (New York: Wiley-Interscience)

[13] Y. Asaki et al., 1998, Radio Science, 33, 1297

[14] E. Middelberg et al., 2005, A\&A, 433, 897 